\title{
Causality, Kramers-Kronig Relations, and Landau Damping
}

\author{
Young Kyung Lim ${ }^{1}$ and Hee J. Lee ${ }^{*}, 2$ \\ ${ }^{1}$ Proton Therapy Center, National Cancer Center, Goyang 410-769, Korea \\ ${ }^{2}$ Department of Physics, Hanyang University, Seoul 133-791, Korea
}

\begin{abstract}
We review the consequence of the causality in electrodynamics. The Kramers-Kronig relations are direct consequence of causality which has two ingredients: no perturbation in the remote past and irrelevance of future susceptibility to the present field. It is shown that the dielectric function obtained from the integration along the Landau contour can be derived from the Kramers-Kronig relations, thus the origin of the collisionless damping in plasmas is traced to the causality. An alternative derivation of the Kramers-Kronig relations based on the causal requirement is presented.
\end{abstract}

PACS numbers: 52.35

Keywords: Causality, Kramers-Kronig relations, Landau damping.

\section{INTRODUCTION}

Causality in electrodynamics means that the response must always follow the cause; the cause cannot be precedent to the effect. For a Fourier component of a wave with phasor

$$
e^{i k x-i \omega t}
$$

the principle of causality can be operationally incorporated by assuming that the frequency $\omega$ has an infinitesimally small positive imaginary part. Then the disturbance at infinitely remote past $(t=-\infty)$ is nil in accordance with the causal notion that the time $t=-\infty$ is necessarily prior to the time of occurrence of any stimulus (cause) which gives rise to the onset of disturbance (effect). The small positive imaginary part $(v)$ of the frequency is called adiabatic switching on of the perturbation at $t=-\infty$. The small positive imaginary frequency $v$ arises mathematically if one solves the plasma equations as an initial value problem via Laplace transform, as Landau demonstrated [1], but it can be simply invoked as an imaginary frequency in accordance with the principle of causality. Adiabatic switching supplies a definition of causality that distinguishes the past from the future. It is well-known that the Kramers-Kronig (K-K) relations are the consequence of the the principle of the causality, as it can be found in the standard text books of electrodynamics.

In this work, we show that Landau damping can be derived from the K-K relations which connects the real and imaginary parts of the plasma dielectric functions. Landau damping which was discovered by Landau in his 1946 paper [1] is interpreted as a consequence of the wave-particle interaction occurring in the kinetic plasma. By deriving

*Address correspondence to this author at the 107-2201 Buyoung Apt. Donong-dong Namyangju-city Kyunggi-do 472-709, Korea;

E-mail: ychjlee@yahoo.com
Landau damping from the $\mathrm{K}-\mathrm{K}$ relations as demonstrated in this work, another interpretation of Landau damping is possible: Landau damping is a consequence of the causality in electrodynamics. This interpretation of the Landau damping provides a new insight in the plasma electrodynamics. It has been accepted that Landau damping can be derived only from the kinetic equation. However, we ought to be able to derive it from the fluid equations as well since the causality prevails regardless of whether we employ kinetic or fluid descriptions of plasma. In this work, we demonstrate that Landau damping can be derived from the fluid equations as well with the aid of the K-K relations (Section III).

In most standard text books of electrodynamics, derivation of the K-K relations utilizes the mathematical fact that the dielectric function is analytic in the upper half $\omega$ plane. Although this analyticity is a consequence of the causality, it is a little abstract to connect the $\mathrm{K}-\mathrm{K}$ realtions to the causality. Here in this work, we derive the K-K relations directly from the causal requirement that the future value of the suscptibility has nothing to do with the present field (Eq. 13 below). Derivation of the K-K relations along this line can be found in earlier work [2]. Here in this work, we use a different mathematical form of the causal requirement, thus enhancing the physical transparency of the relation between the $\mathrm{K}-\mathrm{K}$ relations and the causality.

Incidentally, we mention that the phasor in the expression (1) is compatible with the Fourier transform convention adopted in this work: Fourier transform of a wave function $g(x, t)$ and its inversion are

$$
\begin{aligned}
& g(x, t)=\int_{-\infty}^{\infty} \int_{-\infty}^{\infty} g(k, \omega) e^{i k x-i \omega t} \frac{d k}{2 \pi} \frac{d \omega}{2 \pi} \\
& g(k, \omega)=\int_{-\infty}^{\infty} \int_{-\infty}^{\infty} g(x, t) e^{-i k x+i \omega t} d x d t
\end{aligned}
$$


One who uses different sign in front of the time-phase in the exponential, the frequency $\omega$ should be assumed to have a negative small imaginary frequency.

\section{CAUSALITY AND SUSCEPTIBILITY}

A direct definition of causality is expressed by the statement that susceptibility of future times must be zero. To begin with, we write the Maxwell equation

$\frac{1}{c} \frac{\partial D}{\partial t}=\frac{1}{c} \frac{\partial E}{\partial t}+\frac{4 \pi}{c} J$

where $D$ is the electric displacement, $J$ the plasma current. We integrate Eq. (4) with the initial conditions $D(t)=0$ and $E(t)=0$ at $t=-\infty$ to obtain

$$
D(t)=E(t)+4 \pi \int_{-\infty}^{t} J\left(t^{\prime}\right) d t^{\prime}=E(t)+4 \pi \int_{0}^{\infty} J(t-\tau) d \tau
$$

where we made a change of variable, $t^{\prime}=t-\tau$. In the above equation, $J$ is related with $E$ through material relation. The displacement vector $D(t)$ then represents the response of the medium, or the effect caused by the input $E(t)$. Introducing the material relation

$J(\omega)=\sigma(\omega) E(\omega)=\frac{\omega}{4 \pi i} \chi(\omega) E(\omega)$

in terms of the conductivity $\sigma$ and the susceptibility $\chi$, Eq. (5) can be written as a convolution integral

$$
D(t)=E(t)+\int_{-\infty}^{\infty} \chi\left(t^{\prime}\right) E\left(t-t^{\prime}\right) d t^{\prime}
$$

Causality demands that the kernel $\chi(t)$ in Eq. (6) be zero for $t<0$. This is so because $D$ at present time $t$ should be irrelevant to the values of $E$ at future times. This agrees with the basic idea of causality in natural phenomena, and is vindicated by experience. Thus we impose the condition

$$
\chi(t)=0 \text { for } t<0
$$

This supplies a direct definition of causality. Then, Fourier transforming Eq. (6) gives

$$
\begin{aligned}
& D(\omega)=\varepsilon(\omega) E(\omega), \quad \varepsilon(\omega)=1+\chi(\omega) \\
& \chi(\omega)=\int_{0}^{\infty} \chi(t) e^{i \omega t} d t
\end{aligned}
$$

In the above, we considered implicitly a particular Fourier wave vector component, and the wave vector dependance will be reinstated at appropriate stage.

The half-sided Fourier integral along the positive $t$-axis is analytic in the upper half $\omega$ - plane $\left(\omega_{i}>0 ; \omega=\omega_{r}+i \omega_{i}\right)$ if $\chi(t)$ is square-integrable [3], and $\chi(t)$ is squareintegrable. To present an easier argument for the analyticity of $\varepsilon(\omega)$ in the region $\omega_{i}>0$, let us consider the Fourier integral

$$
\chi(t)=\int_{-\infty}^{\infty} \chi(\omega) e^{-i \omega t} d \omega
$$

In the above integral, we close the contour by encircling the upper half $\omega$-plane when $t<0$. Then, for this integral to be zero, $\chi(\omega)$ should be analytic in the upper half $\omega$ plane. The reverse proposition is also true. To summarize the state of the art so far, the analyticity of $\varepsilon(\omega)$ in the region of $\omega_{i}>0$ is a direct consequence of the causality.

\section{KRAMERS-KRONIG RELATIONS}

In the following, we derive the K-K relations by two different methods. The first method utilizes the analyticity of $\chi(\omega)$ in the upper half $\omega$-plane, while the second method directly uses the causal requirement expressed by Eq. (7).

We review the first method which can be found in standard text books. Let us consider an analytic function $F(\omega)$, analytic in the upper half $\omega$-plane with the property $\lim _{\omega \rightarrow \infty}|F(\omega)|=0$. Then, by Cauchy's integral formula, we can write

$$
F(\omega)=\frac{1}{2 \pi i} \int_{C} \frac{F\left(\omega^{\prime}\right)}{\omega^{\prime}-\omega} d \omega^{\prime}
$$

where $\omega$ is an interior point of the closed contour $C$ which consists of the real $\omega$-axis and the infinite semi-circle in the upper half-plane. Since the contribution from the infinite semi-circle vanishes, the above integral is written

$$
F(\omega)=\frac{1}{2 \pi i} \int_{-\infty}^{\infty} \frac{F\left(\omega^{\prime}\right)}{\omega^{\prime}-\omega} d \omega^{\prime}
$$

This equation reads a complex function $F(\omega)$ of a complex variable $\omega$. Now we let the variable $\omega$ become real by descending the point $\omega$ downward so that it lands on the real axis. Then the integration path in Eq. (10), the path along the real $\omega$-axis, gets deformed in a concave-down shape of infinitesimal semicircle around the singular point $\omega^{\prime}=\omega$. Thus, the integral in Eq. (10) is a sum of the principal value of the integral plus the contribution from the infinitesimal semi-circle. Equating the real and imaginary parts of Eq. (10) respectively to zero, we obtain the K-K relations in the form

$$
\begin{aligned}
& \text { Re } \chi(\omega)=\frac{1}{\pi} P \int_{-\infty}^{\infty} \frac{\operatorname{Im} \chi\left(\omega^{\prime}\right)}{\omega^{\prime}-\omega} d \omega^{\prime} \\
& \operatorname{Im} \chi(\omega)=-\frac{1}{\pi} P \int_{-\infty}^{\infty} \frac{\operatorname{Re} \chi\left(\omega^{\prime}\right)}{\omega^{\prime}-\omega} d \omega^{\prime}
\end{aligned}
$$

The above process in the evaluation of $F(\omega)$ in Eq. (10) when $\omega$ is real is analogous to evaluation of the velocity integral in Eq. (27) along the Landau contour when we have the singularity $v=\omega / k$ on the integration path. In the above equations the symbol $\mathrm{P}$ denotes the principal value.

Next, the second method which directly uses the causal requirement begins with Eq. (6) where we put

$$
\chi(t)=\frac{1}{2}(S(t)+1) \xi(t)
$$

where $S(t)$ is the step function: $S(t)=1$ for $t>0$ and $S(t)=-1$ for $t<0$. The auxiliary function $\xi(t)$ is set to $\chi(t)$ for $t>0$. Then $\chi(t)=\chi(t)$ for $t>0 . \chi(t)$ for $t<0$ can be anything as far as the causality requirement is 
concerned. However, $\xi(t)$ should be somehow related with $\chi(t)$, and we have two possible arrangements: either odd continuation or even continuation into the region $t<0$. For odd (even) continuation, the Fourier transform $\xi(\omega)$ is purely imaginary (real). In the sequel, we only utilize the property of $\xi(\omega)$ being imaginary or real. Different form for the step function $S(t)$ was used by Blundell and Blundell [2] in a similar derivation of the K-K relations.

Now the Fourier transform of $\chi(t)$ in Eq. (13) is the following convolution integral:

$\chi(\omega)=\frac{1}{2 \pi} \int d \omega^{\prime} \xi\left(\omega^{\prime}\right) \frac{1}{2}\left[S\left(\omega-\omega^{\prime}\right)+2 \pi \delta\left(\omega-\omega^{\prime}\right)\right]$

To find the Fourier transform of the step function $S(t)$, we use the identity

$P \int_{-\infty}^{\infty} \frac{e^{i a x}}{x} d x=i \pi$ or $-i \pi$

depending upon $a>0$ or $a<0$. Equation (15) can be proved by performing the contour integration. Therefore we have the following expression for the step function $S(t)$ :

$S(t)=\frac{1}{i \pi} P \int_{-\infty}^{\infty} \frac{e^{i t q}}{q} d q$

The above equation gives for the Fourier transform of the step function in the form

$S(\omega)=\int_{-\infty}^{\infty} S(t) e^{i \omega t} d t=2 i P \frac{1}{\omega}$

Using Eq. (17) in Eq. (14) gives

$\chi(\omega)=\frac{i}{2 \pi} P \int_{-\infty}^{\infty} \frac{\xi\left(\omega^{\prime}\right)}{\omega-\omega^{\prime}} d \omega^{\prime}+\frac{1}{2} \xi(\omega)$

When $\xi(\omega)$ is purely imaginary, we have $\frac{1}{2} \xi(\omega)=i \operatorname{Im} \chi(\omega)$, which gives in turn

$\operatorname{Re} \chi(\omega)=\frac{1}{\pi} P \int_{-\infty}^{\infty} \frac{\operatorname{Im} \chi\left(\omega^{\prime}\right)}{\omega^{\prime}-\omega} d \omega^{\prime}$

When $\xi(\omega)$ is purely real, we have $\frac{1}{2} \xi(\omega)=\operatorname{Re} \chi(\omega)$, which yields in turn

$$
\operatorname{Im} \chi(\omega)=\frac{1}{\pi} P \int_{-\infty}^{\infty} \frac{\operatorname{Re} \chi\left(\omega^{\prime}\right)}{\omega-\omega^{\prime}} d \omega^{\prime}
$$

Equations (19) and (20) are the two K-K relations, which are a pair of Hilbert transforms. Any one of them can be obtained by Hilbert transform from the other. The Hilbert inversion is facilitated by the identity [4]

$\frac{1}{\pi^{2}} P \int_{-\infty}^{\infty} \frac{1}{x^{\prime}-x} \frac{1}{x^{\prime}-x^{\prime \prime}} d x^{\prime}=\delta\left(x-x^{\prime \prime}\right)$

In order to invert Eq. (19), let us multiply both sides by $P \frac{1}{\omega-\omega^{\prime \prime}}$ and integrate $\int_{-\infty}^{\infty} d \omega(\cdots)$ :
$P \int_{-\infty}^{\infty} \frac{\operatorname{Re} \chi(\omega)}{\omega-\omega^{\prime \prime}} d \omega=\frac{1}{\pi} P \int_{-\infty}^{\infty} \frac{d \omega}{\omega-\omega^{\prime \prime}} \int_{-\infty}^{\infty} \frac{\operatorname{Im} \chi\left(\omega^{\prime}\right)}{\omega^{\prime}-\omega} d \omega^{\prime}$

Upon using formula (21) the right hand side of the above equation becomes $-\pi \operatorname{Im} \chi\left(\omega^{\prime \prime}\right)$. So we obtain

$\operatorname{Im} \chi(\omega)=-\frac{1}{\pi} P \int_{-\infty}^{\infty} \frac{\operatorname{Re} \chi\left(\omega^{\prime}\right)}{\omega^{\prime}-\omega} d \omega^{\prime}$

\section{LANDAU DAMPING}

As a concrete example of the K-K relations, we consider a one-dimensional Vlasov-Poisson plasma which is governed by the following linearized equations:

$\frac{\partial}{\partial t} g(x, v, t)+v \frac{\partial g}{\partial x}-\frac{e}{m} E(x, t) \frac{d g_{0}(v)}{d v}=0$

$\frac{\partial}{\partial x} E(x, t)=-4 \pi e \int_{-\infty}^{\infty} d v g(x, v, t)$

where $g(x, v, t)$ is the perturbed electron distribution function, $g_{0}(v)$ is the zero order equilibrium electron distribution function, and the ions are assumed to form the uniform neutralizing background. We Fourier transform Eqs. (23) and (24) by performing the integral $\iint e^{-i k x+i \omega t}(\cdots) d x d t$. Assuming that $\omega$ has a small positive imaginary part and using the causal boundary condition $g(x, v, t=-\infty)=0$ give

$$
\begin{aligned}
& g(k, v, \omega)=\frac{e}{m} \frac{d g_{0}}{d v} \frac{i}{\omega-k v} E(k, \omega) \\
& i k E(k, \omega)=-4 \pi e \int_{-\infty}^{\infty} d v g(k, v, \omega)
\end{aligned}
$$

The dielectric function is obtained from the above equations

$\varepsilon(k, \omega)=1+\chi(\omega, k)=1+\frac{\omega_{p e}^{2}}{k} \int_{-\infty}^{\infty} d v \frac{\frac{d g_{0}}{d v}}{\omega-k v}$

where $\omega_{p e}$ is the electron plasma frequency. We show that $\chi(\omega, k)$ in Eq. (27) satisfies the K-K relations. The real part of $\chi(k, \omega)$ is given by the principal value:

$\operatorname{Re} \chi(k, \omega)=-P \int_{-\infty}^{\infty} d v \frac{\frac{d G_{0}(v)}{d v}}{v-\frac{\omega}{k}}$

where $G_{0}(v)=\frac{\omega_{p e}^{2}}{k^{2}} g_{0}(v)$. The imaginary part of $\chi(k, \omega)$ is obtained by using Eq. (28) in Eq. (20):

$$
\begin{aligned}
& \operatorname{Im} \chi(k, \omega)=\frac{1}{\pi} P \int_{-\infty}^{\infty} \frac{\operatorname{Re} \chi\left(\omega^{\prime}, k\right)}{\omega-\omega^{\prime}} d \omega^{\prime} \\
& =\frac{1}{\pi} P \int_{-\infty}^{\infty} \frac{d \omega^{\prime}}{\omega^{\prime}-\omega} P \int_{-\infty}^{\infty} d v \frac{\frac{d G_{0}(v)}{d v}}{v-\frac{\omega^{\prime}}{k}}
\end{aligned}
$$


$=-\frac{1}{\pi} \int_{-\infty}^{\infty} d v \frac{d G_{0}(v)}{d v} P \int_{-\infty}^{\infty} d \xi \frac{1}{\xi-v} \frac{1}{\xi-\omega / k} \quad\left(\xi=\omega^{\prime} / k\right)$

Upon using Eq. (21) the above expression becomes

$\operatorname{Im} \varepsilon(k, \omega)=-\pi\left[\frac{d G_{0}}{d v}\right]_{v=\frac{\omega}{k}}$

This result is completely equivalent to calculating the velocity integral in Eq. (27) along the Landau contour. Reversely, if Eq. (29) is substituted into Eq. (19), we recover Eq. (28).

As another example of application of K-K relations, we shall derive Landau damping by means of fluid equations. The plasma under consideration which obeys the kinetic equations, Eqs. (23) and (24), is considered as a group of beams [5]; each beam with the beam velocity $v_{0}$ is described by fluid equations

$\frac{\partial v}{\partial t}+v_{0} \frac{\partial v}{\partial x}=-\frac{e}{m} E(x, t)$

$\frac{\partial n}{\partial t}+N\left(v_{0}\right) \frac{\partial v}{\partial x}+v_{0} \frac{\partial n}{\partial x}=0$

$i k E(k, \omega)=-4 \pi e n(k, \omega)$

where $v$ and $n$ are respectively perturbed velocity and perturbed density of the beam whose zero order velocity is $v_{0} . N\left(v_{0}\right)$ is the equilibrium zero order number density of $v_{0}$-beam. In terms of the Fourier variables, Eqs. (30) and (31) are written

$i\left(-\omega+k v_{0}\right) v=-\frac{e}{m} E(k, \omega)$

$\left(-\omega+k v_{0}\right) n+k N\left(v_{0}\right) v=0$

The last two equations yield

$n=-i \frac{e}{m} \frac{k N\left(v_{0}\right)}{\left(\omega-k v_{0}\right)^{2}} E$

The polarization $P=-\frac{E}{4 \pi}$ is obtained after using Eqs. (32) and (35)

$$
P=-\frac{e^{2}}{m} \frac{N\left(v_{0}\right)}{\left(\omega-k v_{0}\right)^{2}} E
$$

Therefore the dielectric function of $v_{0}$-beam is obtained from $E+4 \pi P=\varepsilon_{b} E$ :

$\varepsilon_{b}(k, \omega)=1-\frac{4 \pi e^{2}}{m} \frac{N\left(v_{0}\right)}{\left(\omega-k v_{0}\right)^{2}}$

The real part of $\varepsilon_{b}$ is the principal value:

$$
\operatorname{Re} \varepsilon_{b}(k, \omega)=1-\frac{4 \pi e^{2} N\left(v_{0}\right)}{m} P \frac{1}{\left(\omega-k v_{0}\right)^{2}}
$$

The imaginary part of $\varepsilon_{b}$ is obtained from the K-K relation by evaluating the integral

$$
\begin{aligned}
& \operatorname{Im} \varepsilon_{b}(k, \omega)=-\frac{1}{\pi} P \int \frac{\operatorname{Re}\left[\varepsilon_{b}\left(k, \omega^{\prime}\right)-1\right]}{\omega^{\prime}-\omega} d \omega^{\prime} \\
& =\frac{1}{\pi} \frac{4 \pi e^{2} N\left(v_{0}\right)}{m} P \int_{-\infty}^{\infty} \frac{d \omega^{\prime}}{\omega^{\prime}-\omega} P \frac{1}{\left(\omega^{\prime}-k v_{0}\right)^{2}}
\end{aligned}
$$

Here we use the relation

$P \frac{1}{\left(\omega-k v_{0}\right)^{2}}=-\frac{1}{k^{2}} \frac{\partial}{\partial v_{0}} P \frac{1}{v_{0}-\frac{\omega}{k}}$

Then we obtain with the aid of Eq. (21)

$\operatorname{Im} \varepsilon_{b}(k, \omega)=\pi \frac{4 \pi e^{2} N\left(v_{0}\right)}{m k} \frac{\partial}{\partial v_{0}} \delta\left(\omega-k v_{0}\right)$

The plasma susceptibility is obtained by summing all the beam susceptibilities since the susceptibility is additive. Let the distribution of beams be $f\left(v_{0}\right)$. Putting $N\left(v_{0}\right)=f\left(v_{0}\right) d v_{0}$ and integrating over the beams, we obtain

$\operatorname{Re} \varepsilon(k, \omega)=1+\frac{\omega_{p e}^{2}}{k^{2}} P \int_{-\infty}^{\infty} \frac{\partial}{\partial v_{0}} \frac{1}{v_{0}-\frac{\omega}{k}} f\left(v_{0}\right) d v_{0}$

$=1+\frac{\omega_{p e}^{2}}{k} P \int_{-\infty}^{\infty} \frac{\frac{d f\left(v_{0}\right)}{d v_{0}}}{\omega-k v_{0}} d v_{0}$

$\operatorname{Im} \varepsilon(k, \omega)=-\pi \frac{\omega_{p e}^{2}}{k^{2}}\left[\frac{d f\left(v_{0}\right)}{d v_{0}}\right]_{v_{0}=\frac{\omega}{k}}$

The dielectric function as obtained by Eqs. (40) and (41) entirely agrees with the kinetic theory results calculated according to Landau's prescription.

\section{DISCUSSION}

Finally we show that separation of the real and imaginary parts of $\varepsilon_{b}$ in Eq. (37) can be done without using the K-K relations; it can be done by putting $\omega \rightarrow \omega+i v$ in correspondence to the adiabatic switching. We have

$$
\frac{1}{\left(\omega+i v-k v_{0}\right)^{2}}=\frac{1}{\left(\omega-k v_{0}\right)^{2}+v^{2}}-i \frac{2 v\left(\omega-k v_{0}\right)}{\left[\left(\omega-k v_{0}\right)^{2}+v^{2}\right]^{2}}
$$

Now we let $v \rightarrow 0$. Then the real part of this expression is $P \frac{1}{\left(\omega-k v_{0}\right)^{2}}$, the principal value. The imaginary part is $\frac{-2 i v\left(\omega-k v_{0}\right)}{\left[\left(\omega-k v_{0}\right)^{2}+v^{2}\right]^{2}}=i \pi \frac{\partial}{\partial \omega^{\prime}} \frac{v}{\pi\left(\omega^{\prime 2}+v^{2}\right)} \quad\left(\omega^{\prime}=\omega-k v_{0}\right)$

Letting $v \rightarrow 0$, the above expression becomes $i \pi \frac{\partial}{\partial \omega^{\prime}} \delta\left(\omega^{\prime}\right)=-i \pi \frac{1}{k} \frac{\partial}{\partial v_{0}} \delta\left(\omega-k v_{0}\right)$ 
Using the above result Eq. (39) is recovered. This exercise shows that use of Plemelj's formula is a way of implementing the causal requirement. As it is well-known Plemelj's formula is equivalent to the Landau contour.

In conclusion, the principle of causality was enforced by two mathematical apparatus, namely, the adiabatic switching $(\omega \rightarrow \omega+i v)$ and Eq. (13) which definitely specifies $\chi(t<0)=0$. The two mathematical implementations of the causal requirement resulted in the K-K relations, and equivalently the dielectric function calculated along the Landau contour.

In summary, we showed that Landau damping can be derived from the K-K relations which are direct consequence of the causality. Thus it appears to be possible to have a new interpretation of Landau damping: its fundamental reason can be traced to the causality. It has been usually accepted that derivation of Landau damping requires kinetic equation which explicitly comprises the wave-particle interaction in plasma. Our fluid derivation of Landau damping further supports this new interpretation since the causality is operative regardless of whether we adopt kinetic or fluid model of plasma.

\section{CONFLICT OF INTEREST}

The authors confirm that this article content has no conflict of interest.

\section{ACKNOWLEDGEMENTS}

Declared none.

\section{REFERENCES}

[1] Landau L. On the vibration of the electronic plasma. J Phys 1946; 10: 25-34.

[2] Blundell SJ, Blundell KM. Concepts in thermal physics. New York: Oxford University Press 2010.

[3] Titchmarsh EC. Introduction to the theory of fourier integrals. Oxford: Clarendon Press 1937.

[4] Lokenath D, Mikusinski P. Introduction to hilbert spaces with applications. San Diego: Academic Press 1999; Van Kampen NG, Felderhof BU. Theoretical methods in plasma physics. New york: John Wiley 1967.

[5] Bohm D, Gross EP. Theory of plasma oscillations. A: Origin of medium-like behavior B: Excitation and damping of oscillations. Phys Rev 1949: 75; 1851-76. 